\section{Case Reports in Neurology}

This article is licensed under the Creative Commons Attribution-NonCommercial 4.0 International License (CC BY-NC) (http://www.karger.com/Services/OpenAccessLicense). Usage and distribution for commercial purposes requires written permission.

\title{
Herniated Proximal Marker of the Stent into the Meningohypophyseal Trunk after Stent-Assisted Coil Embolization for Intracranial Aneurysms
}

\author{
Won-Bae Seung \\ Department of Neurosurgery, Dongguk University Gyeongju Hospital, Dongguk University \\ College of Medicine, Gyeongju, Republic of Korea
}

\section{Keywords}

Meningohypophyseal trunk · Proximal marker · Solitaire ${ }^{\mathrm{TM}} \mathrm{AB}$ Neurovascular Remodeling Device

\section{Abstract}

The Solitaire ${ }^{T M}$ AB Neurovascular Remodeling Device (ev3, Irvine, CA, USA) is used to retain coils within an aneurysm, reducing the risk of embolic complications from coil herniation into the parent artery. Stents are deployed after confirming the optimal position of the stent markers across the aneurysm to avoid entry into perforators or branching arteries. Stent marker position is very important to prevent perforating or branching artery infarction. We performed stent-assisted coil embolization using the Solitaire $A B$ stent to treat 2 aneurysms simultaneously. After successful coil embolization, we detached the Solitaire stent in the usual manner. However, the proximal stent marker, which was located at the horizontal segment of the cavernous internal carotid artery, moved into the meningohypophyseal trunk and occluded it. Although the distal markers were positioned optimally, we did not expect the proximal marker to be in the position where it was located. Fortunately, cranial nerve dysfunctions and pituitary deficiency did not develop. It is important to prevent ischemic injury by occlusion of the perforators or branching vessels that can be caused by malpositioned stent markers. We consider where the proximal marker of the stent might be located after detachment. 


\section{Introduction}

Stent-assisted coil embolization is performed worldwide and is both safe and efficacious for treating wide-necked and complex intracranial aneurysms. During the last decade, several neurovascular remodeling devices were widely used and generally accepted for the endovascular treatment of intracranial aneurysms. Among the available self-expandable intracranial stents, the Solitaire ${ }^{\mathrm{TM}}$ AB Neurovascular Remodeling Device (ev3, Irvine, CA, USA) has desirable characteristics. It can be more precisely placed and deployed compared to other stents before detachment. However, unexpected events can occur despite optimal stent position. We report one case of proximal marker of the Solitaire AB stent herniated into the meningohypophyseal trunk (MHT) after stent-assisted coil embolization for ophthalmic and paraclinoid aneurysms.

\section{Case Report}

A 53-year-old woman was found to have dual aneurysms located at the ophthalmic artery and paraclinoid area of the internal carotid artery (ICA). We performed stent-assisted coil embolization using the jailing technique for 2 aneurysms one by one in a single procedure under general anesthesia. This procedure was successful, resulting in complete occlusion of aneurysmal sacs, and the Solitaire AB stent was detached by the electrical detachment system (Fig. 1a). Final cerebral angiography revealed that the stent was deployed in optimal position across the neck of aneurysm and the distal markers were placed optimally without entering into branching arteries or perforators. However, we found that the proximal marker moved from the upper part of the cavernous segment of the ICA to the MHT after detachment of the stent (Fig. 1b). We checked the patient's condition with postoperative magnetic resonance images including diffusion-weighted images and hormonal studies. The patient has no complications or other symptoms.

\section{Discussion}

The Solitaire ${ }^{\mathrm{TM}} \mathrm{AB}$ Neurovascular Remodeling Device is a laser-cut, self-expanding nitinol device [1]. The distinctive characteristic of this device is full retrievability and redeployment to achieve optimal alignment across the neck of the aneurysm until it is electrically detached from the push wire [1]. The Solitaire AB stent has 3 or 4 distal markers and 1 proximal marker and consists of usable length and retrieval zone. The proximal marker is thicker than distal markers. The positions of distal markers are very important, because if they are herniated to a perforator or side branch, they may occlude, and cerebral infarction may occur. Commonly, when the stent is deployed across the aneurysmal neck, operators try to optimally place distal markers to avoid jailing of perforators by stent markers. However, operators cannot predict the position of the proximal marker after full deployment. After the stent is fully deployed, it is not possible to reposition. In our case, we applied the $6 \times 30 \mathrm{~mm}$ Solitaire AB stent to cover the aneurysmal necks of the ophthalmic artery and paraclinoid ICA simultaneously. The Solitaire $\mathrm{AB}$ stent was deployed from the start of the proximal part of the posterior communicating artery to the midportion of the cavernous segment of the ICA, and the 2 aneurysmal necks were completely covered by the usable part of the Solitaire AB stent. A balanced combination technique of the stent delivery microwire push and delivery microcatheter pull-back 
was used to optimize the stent deployment and minimize the stent malapposition [2]. However, the proximal marker of the stent moved proximally after detachment and entered into the MHT because the tension of the stent delivery microwire was released after detachment. Lateral arterial phase imaging of the cerebral angiography demonstrated a pituitary brush before detachment of the stent. However, the pituitary brush disappeared because MHT was occluded by the proximal marker after detachment of the stent.

The MHT, originating from the proximal cavernous segment of the ICA, may be a single trunk or a collection of vessels. The classic main 3 branches of the MHT are the tentorial marginal artery, the dorsal meningeal artery, and the inferior hypophyseal artery [3]. MHT vessels supply the cranial nerves III, IV, V, and VI, the pituitary gland, portions of the clivus, tentorium cerebelli, and the adjacent dura [3]. MHT occlusion presents a risk of ischemic injury to cranial nerves and the pituitary gland. However, some research reports that embolization or occlusion of these arteries was not accompanied by cranial nerve dysfunction [3]. In our case, the MHT was occluded by the proximal marker of the stent, but the patient has no cranial nerve dysfunctions and not any other clinical and laboratory abnormalities.

\section{Conclusion}

The Solitaire AB stent has 4 distal and 1 proximal marker sized $6 \times 30 \mathrm{~mm}$. It is important to prevent ischemic injury by occlusion of the perforators or branching vessels resulting from malpositioned stent markers. In our case, we could not anticipate the change of position of the proximal marker because of backward migration after electrical detachment. We considered where the proximal marker would be located after detachment.

\section{Statement of Ethics}

The authors have no ethical conflicts.

\section{Disclosure Statement}

The authors have no conflicts of interest to disclose.

\section{References}

1 Clajus C, Sychra V, Strasilla C, Klisch J. Stent-assisted coil embolization of intracranial aneurysms using the Solitaire ${ }^{\mathrm{TM}}$ AB Neurovascular Remodeling Device: initial and midterm follow-up results. Neuroradiology. 2013 May;55(5):629-38.

2 Heller RS, Malek AM. Delivery technique plays an important role in determining vessel wall apposition of the Enterprise self-expanding intracranial stent. J Neurointerv Surg. 2011 Dec;3(4):340-3.

3 Robinson DH, Song JK, Eskridge JM. Embolization of meningohypophyseal and inferolateral branches of the cavernous internal carotid artery. AJNR Am J Neuroradiol. 1999 Jun-Jul;20(6):1061-7. 


\section{Case Reports in Neurology}
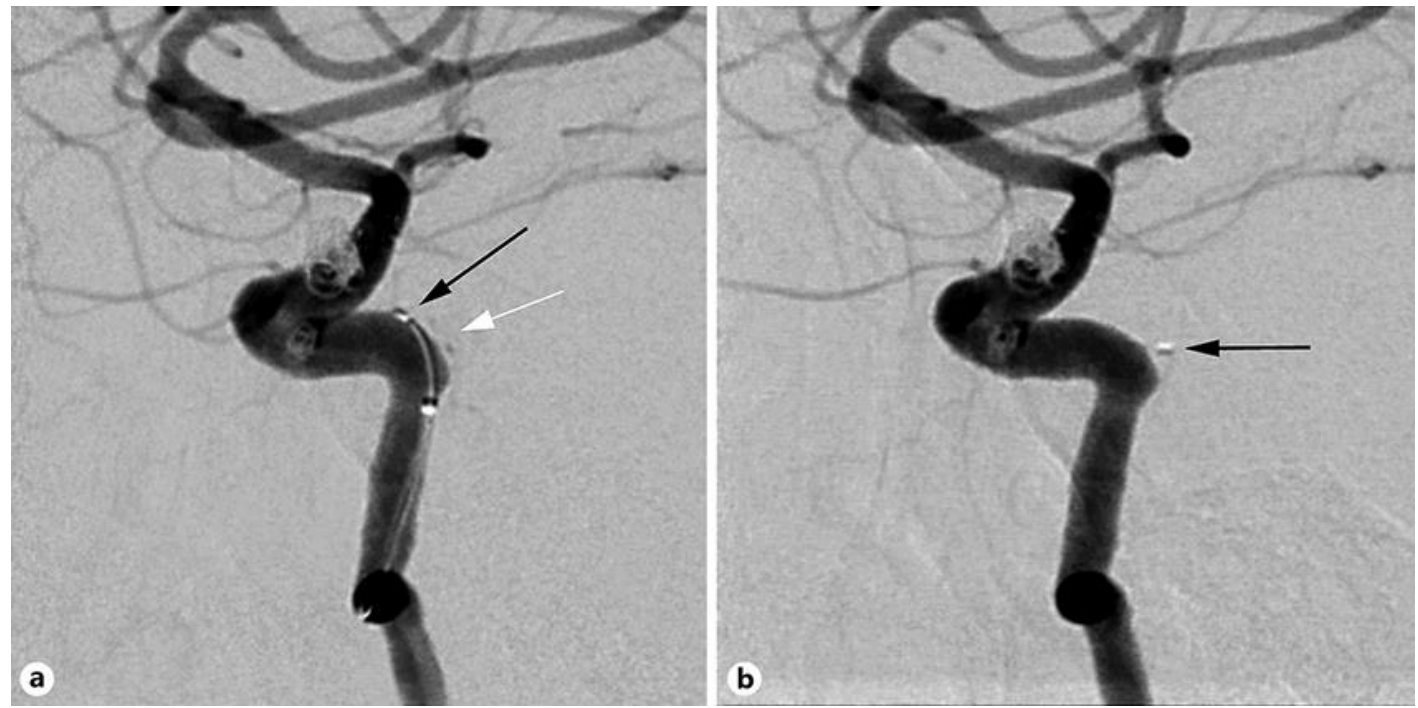

Fig. 1. A 53-year-old woman was found to have dual aneurysms located at the ophthalmic artery and paraclinoid area of the internal carotid artery (ICA). We performed stent-assisted coil embolization for treating 2 aneurysms in ophthalmic artery and paraclinoid area of the ICA simultaneously (a). After detachment of the stent, we identified that the proximal marker bounced and moved into the meningohypophyseal trunk (b). The black arrow indicates the proximal marker of the stent and the white arrow indicates the meningohypophyseal trunk. 